\title{
Isogeometric Analysis for the numerical simulation of rubber structures
}

\author{
S. Lejeunes \& D. Eyheramendy \\ Aix-Marseille Univ, CNRS, Centrale Marseille, LMA UMR 7031, F-13402 Marseille Cedex 20, France
}

\begin{abstract}
In this work, we propose to discuss about the interrest of the Isogeometric Analysis as a computational framework for the numerical simulation of rubber structures with a multi-field approach. We show that we can construct stable approximations for hyperelastic problems using the same approach as that used for finite-elements. We also show that the extension to thermo-mechanical problems is very simple. Using simple numerical examples and the same variationnal formulation, we compare the numerical performance of IGA and FE.
\end{abstract}

\section{INTRODUCTION}

Iso-geometric analysis (IGA), introduced by Hughes and co-authors can be seen as a B-Spline or a NURBS based finite element, see Hughes, Cottrell, \& Bazilevs 2005, Hughes, Cottrell, \& Bazilevs 2009. The first main consequence of this change is that the space discretization is not made in the physical space but in a parametric space ${ }^{1}$ that is much simpler. The properties of these new approximation functions make it possible to exactly describe complex geometries (without any approximation). Compared to Lagrange polynomials, we have an additional degree of freedom with these functions since we can control the continuity order at the level of the nodes of the mesh, independently of the order of the functions and the mesh size. Finally, the definition of these functions by recurrence makes it possible to build very simply high order basis. All these properties have led to the rapid use of the isogeometric analysis for many applications and many authors have already shown that it has very good numerical qualities. The main limitation of its extension in commercial codes is, at present, the lack of links between CAD tools that only describe the contours of an object and the simulation world that needs to use the inside of objects (3D volume description).

Despite its great success, Isogeometric analysis is currently not widely used in the context of incompressible or weakly compressible elasticity and therefore there are very few applications for rubber based structures. Nevertheless, we can note the work of

\footnotetext{
${ }^{1}$ Compared to classical finite elements, there are three spaces in an IGA formulation: physical space (geometry of the object), parametric space (support for the construction of functions), reference space (for numerical integration by quadrature schemes)
}

Buffa \& Sangalli 2010, Nielsen, Gersborg, Gravesen, \& Pedersen 2011, Elguedj \& Hughes 2014, Kadapa, Dettmer, \& Perić 2016 for linear (small strain) and non-linear (finite strain) problems with different types of formulations. For this type of problems, the question of the numerical stability is central and o can find different approaches in the literature that account of numerical stability, e.g.: enhanced-strain, selectivereduced integration, specific multi-field methods. In Lei Zhang's phd thesis (Zhang 2016), we explored different mixed formulations $(\mathbf{u}, p)$ for nearly incompressible linear and non-linear elasticity by playing on:

- the order of the approximation of each field (kinematics and pressure)

- the order of continuity

- the sub-discretization of one or several fields

We were able to find stable approximations in small strain (which correspond to the proposals of Nielsen, Gersborg, Gravesen, \& Pedersen 2011, Buffa \& Sangalli 2010) but which can become unstable extended to finite-strain. We have also proposed a stable approximation at finite strain that has not been discussed yet in the literature to our knowledge. Using the same formalism we have also looked at coupled thermomechanical problems in the context of entropic elasticity for which, to our knowledge, there exhists no work based on isogeometric analysis. With the help of some numerical examples, we show that IGA can be of interest in the context of mixed or multi-physics formulations. 


\section{MIXED FORMULATIONS}

Mixed variational formulations are here established using the formalim already developed in (Lejeunes \& Eyheramendy 2018). The definition of a hybrid free energy, $\varphi(\overline{\mathbf{F}}, \Theta, q)$ where $q$ is a stress (pressure-like) variable, allows to obtain straightforwardly mixed formulations for nearly-incompressible hyperelasticity or coupled thermomechanical elasticity ${ }^{2}$. These mixed formulations are very closed from those obtained with the Hellinger-Reissner variational principle. This principle is based on the same idea: using a partial Legendre transformation of the strain energy such as to consider a scalar stress variable (pressure) instead of a scalar strain variable (volume variation).

\section{$2.1 \quad$ Nearly-incompressible hyperelasticity}

We adopt the so-called volumic/isochoric split of the deformation gradient (see Flory 1961):

$\mathbf{F}(\mathbf{X}, t)=\nabla_{\mathbf{X}} \mathbf{u}(\mathbf{X}, t)+\mathbf{1}=\overline{\mathbf{F}}\left(J^{1 / 3} \mathbf{1}\right)$

where $J=\operatorname{det}(\mathbf{F})$ is the volume variation, $\mathbf{1}$ is the identity tensor. The first Piola-Kirchhoff stress, $\Pi$, can be expressed from a hybrid energy $\varphi(\overline{\mathbf{F}}, q)$ where $q$ in this case is equivalent to the hydrostatic stress if we assume an elastic behavior. The choice of a splitting of the free energy such as $\varphi(\mathbf{F}, q)=\varphi_{\text {iso }}(\overline{\mathbf{F}})+$ $g(q)$ leads to the definition of a compressibility law (constitutive equation for $q$ ). Taking the weak form of the static stress equilibrium equation and the compressibility law, we can obtain the following variational system:

Find $(\mathbf{u}, q) \in\left(\mathcal{U} \times L^{2}\left(\Omega_{0}\right)\right)$

such as $\forall(\delta \mathbf{u}, \delta q) \in\left(\mathcal{U}^{0} \times L^{2}\left(\Omega_{0}\right)\right)$ :

$$
\begin{aligned}
& \int_{\Omega_{0}}(\overline{\mathbf{\Pi}}:\left.\mathbb{P}_{\mathbf{F}}+q J \mathbf{F}^{-\mathrm{T}}\right): \nabla_{\mathbf{X}} \delta \mathbf{u} d \Omega_{0}-\int_{\Omega_{0}} \mathbf{f} \delta \mathbf{u} d \Omega_{0} \\
&-\int_{\partial \Omega_{f_{0}}} \mathbf{t} \delta \mathbf{u} d S=0
\end{aligned}
$$$$
\int_{\Omega_{0}}\left((J-1)-\frac{\partial g}{\partial q}\right) \delta q d \Omega_{0}=0
$$

with $=\partial \varphi_{i s o} / \partial \overline{\mathbf{F}}, \mathbb{P}_{\mathbf{F}}$ is the deviatoric operator in mixed configuration, $g$ is a compressibility potential. The spaces $\mathcal{U}, \mathcal{U}^{0}$ are such that $\mathcal{U}^{0}=$ $\left\{\delta \mathbf{u} \in\left(H^{1}\left(\Omega_{0}\right)\right)^{n}, \delta \mathbf{u}=0\right.$ on $\left.\partial \Omega_{u_{d}}\right\}, \mathcal{U}=\{\mathbf{u} \in$ $\left(H^{1}\left(\Omega_{0}\right)\right)^{n}, \mathbf{u}=\mathbf{u}_{0}$ on $\left.\partial \Omega_{u_{d}}\right\}$, with $n$ the dimension of the physical space.

\footnotetext{
${ }^{2}$ Other non-linear effects like viscoelasticty can be taken into account without any difficulties
}

\subsection{Thermo-mechanical coupling: modified entropic elasticity}

For thermoelastic problems, we adopt a formulation that is similar to the modified entropic theory such as presented in Miehe 1995. We introduce a supplementary term in the splitting of the deformation gradient such as to account of a thermal deformation (dillatation). Equation (1) becomes:

$\mathbf{F}(\mathbf{X}, t)=\overline{\mathbf{F}}\left(J_{m}^{1 / 3} \mathbf{1}\right)\left(J_{\Theta}^{1 / 3} \mathbf{1}\right)$

where $J_{\Theta}=1+\alpha\left(\Theta-\Theta_{0}\right)$ is the thermal dilatation and $J_{m}=J / J_{\Theta}$ is the mechanical volume variation ( $\alpha$ is the thermal expension coefficient). Taking the static stress equilibirum, the energy conservation and the compressibility law, we can obtain the following variational system (in this cas $q$ is not strictly equals to the hydrostatic pressure):

Find $(\mathbf{u}, \Theta, q) \in\left(\mathcal{U} \times \mathcal{T} \times L^{2}\left(\Omega_{0}\right)\right)$

such as $\forall(\delta \mathbf{u}, \delta \Theta, \delta q) \in\left(\mathcal{U}^{0} \times \mathcal{T}^{0} \times L^{2}\left(\Omega_{0}\right)\right)$ :

$$
\begin{aligned}
\int_{\Omega_{0}}\left(\overline{\mathbf{\Pi}}: \mathbb{P}_{\mathbf{F}}+q \frac{J}{J_{\Theta}} \mathbf{F}^{-\mathrm{T}}\right): \nabla_{\mathbf{X}} \delta \mathbf{u} d \Omega_{0}-\int_{\Omega_{0}} \mathbf{f} \delta \mathbf{u} d \Omega_{0} \\
-\int_{\partial \Omega_{f_{0}}} \mathbf{t} \delta \mathbf{u} d S=0
\end{aligned}
$$$$
\int_{\Omega_{0}} \rho_{0} C_{p} \dot{\Theta} \delta \Theta d \Omega_{0}-\int_{\Omega_{0}}\left(J l_{m}+J l_{q}+\rho_{0} r\right) \delta \Theta d \Omega_{0}
$$

$$
-\int_{\Omega_{0}} J \mathbf{Q} \nabla_{\mathbf{X}} \delta \Theta d \Omega_{0}+\int_{\partial \Omega_{Q_{0}}} \mathbf{Q}_{h} \mathbf{N} \delta \Theta d S=0
$$

$$
\int_{\Omega_{0}}\left(\left(J_{m}-1\right)-\frac{\partial g}{\partial q}\right) \delta q d \Omega_{0}=0
$$

where $C_{p}$ is the heat capacity at constant pressure, $\mathbf{Q}$ is the heat flux in lagrangian configuration, $l_{m}$ and $l_{q}$ are thermoelastic coupling terms, $r$ is an external volumic heat supply, $\mathrm{Q}_{h}$ is the heat flux that is prescribed on the boundary $\partial \Omega_{Q_{0}}$. The expression of $C_{p}, l_{m}, l_{q}$ are given in Lejeunes \& Eyheramendy 2018.

The spaces $\mathcal{T}, \mathcal{T}^{0}$ are such that $\mathcal{T}^{0}=$ $\left\{\delta \Theta \in\left(H^{1}\left(\Omega_{0}\right)\right)^{n}, \delta \Theta=0\right.$ on $\left.\partial \Omega_{\Theta_{0}}\right\} \quad$ and $\mathcal{T}=\left\{\Theta \in\left(H^{1}\left(\Omega_{0}\right)\right)^{n}, \Theta=\Theta_{d}\right.$ on $\left.\partial \Omega_{\Theta_{0}}\right\}$, with $n$ the dimension of the physical space.

Initial thermal and mechanical conditions must be prescribed to complete the previous system. Before linearizing the previous nonl-linear variational form with a Newton-Raphson scheme, we adopt a backward Euler time discretisation of the equations. We 
therefore obtain a fully coupled system (and a nonsymetric tangent) to account of strongly coupled thermomechanical cases. Using the approximation $\dot{\Theta}=$ $\left(\Theta^{n+1}-\Theta^{n}\right) / \Delta t$, the system of eqs. (4) can be written as follows on a time interval $\left[t_{n}, t_{n+1}\right]$ :

Find $\left(\mathbf{u}^{n+1}, \Theta^{n+1}, q^{n+1}\right)$, knowing $\left(\mathbf{u}^{n}, \Theta^{n}, q^{n}\right)$

such that $\forall(\delta \mathbf{u}, \delta \Theta, \delta q)$ :

$$
\begin{aligned}
& \int_{\Omega_{0}}\left(\overline{\mathbf{\Pi}}^{n+1}: \mathbb{P}_{\mathbf{F}^{n+1}}+q^{n+1} \frac{J^{n+1}}{J_{\Theta^{n+1}}} \mathbf{F}^{n+1^{-\mathrm{T}}}\right): \nabla_{\mathbf{X}} \delta \mathbf{u}_{h} d \Omega_{e} \\
& -\int_{\Omega_{0}} \mathbf{f}^{n+1} \delta \mathbf{u}_{h} d \Omega_{e}-\int_{\partial \Omega_{f_{0}}} \mathbf{t}^{n+1} \delta \mathbf{u} d S=0
\end{aligned}
$$$$
\int_{\Omega_{0}} \rho_{0} \frac{C_{p}^{n+1}}{\Delta t}\left(\Theta^{n+1}-\Theta^{n}\right) \delta \Theta d \Omega_{0}-\int_{\Omega_{0}}\left(J^{n+1} \phi_{m}^{n+1}\right.
$$$$
\left.+J^{n+1} l_{m}^{n+1}+J^{n+1} l_{q}^{n+1}+\rho_{0} r^{n+1}\right) \delta \Theta d \Omega_{0}-
$$$$
\int_{\Omega_{0}} J^{n+1} \mathbf{Q}^{n+1} \nabla_{\mathbf{X}} \delta \Theta d \Omega_{0}+\int_{\partial \Omega_{Q_{0}}} \mathbf{Q}_{h}^{n+1} \mathbf{N} \delta \Theta d S_{0}=0
$$$$
\int_{\Omega_{0}}\left(\left(J_{m}^{n+1}-1\right)+\left(\frac{\partial g}{\partial q}\right)^{n+1}\right) \delta q d \Omega_{0}=0
$$

\section{SOME ELEMENTS ABOUT THE ISOGEOMETRIC ANALYSIS IMPLEMENTATION}

Figure 1 shows a synthetic comparison of the construction of approximation spaces with finite elements and isogeometric analysis. With the IGA, the mesh is carried out in one (or more) parametric space $(\mathrm{s})^{3}$. From this mesh, we can construct the following approximations on a patch (2D case with $(\mathbf{u}, q))$ :

$$
\begin{aligned}
& \mathbf{u}^{h}(x, y)=\sum_{i} \sum_{j} R_{i}^{p}(\xi) R_{j}^{p}(\eta) B_{i j}^{u} \\
& q^{h}(x, y)=\sum_{i} \sum_{j} R_{i}^{r}(\xi) R_{j}^{r}(\eta) B_{i j}^{q}
\end{aligned}
$$

the exponants $p$ and $r$ are the orders of the NURBS, $R_{j}$ et $R_{i}$ functions, for each field. These functions are

\footnotetext{
${ }^{3}$ With IGA, there is also the notion of patchs: in many situations physical space is described by more than one patch or approximation space. Each patch has its own parametric space and therefore its own parametric mesh. Enforcing continuity of unknown fields between patch can be done with specific methods.
}

built in a recursive way. The parameters $B_{i j}$ are the degrees of freedom that can be weighted to define conical geometries for instance. By repetiting nodes in the mesh, we control the order of continuity at mesh nodes. A NURBS function is interpolating if you repeat $p+1$ times the nodes at the boundary of the parametric space or $p$ times inside the parametric space. We have a structure similar to that of finite element, but with some major differences, such as:

- due to the use of rational polynomials (NURBS), the numerical integration by quadrature schemes is not exact and requires more quadrature points than for finite element ${ }^{4}$.

- the geometric support of NURBS functions does not correspond to an element of the parametric mesh and therefore matrices are not as sparse as for finite elements

In the case of multifield formulations, we can play with: i) the approximation order of each field, ii) the continuity order of each field, iii) the parametric mesh that can be different for each field (subdiscretisation). In the following, to define the approximation space for each field, we adopt the notation: $U p_{\text {sub }}^{\text {cont }} P q_{\text {sub }}^{\text {cont }}$ where $p$ and $q$ are the approximation orders, the $s u b$ indices give information on the mesh subdivision ( $s u b=1$, no subdivision) and cont gives the multiplicity of internal nodes and controls the continuity order of each field (cont $=1$ for a multiplicity of 1 and a continuity $C_{p}$, or $C_{q}$, of the field in the mesh). The number of degrees of freedom is therefore determined by the parameters $p$ (or $q$ ), cont, sub, and the number of nodes per direction of the parametric space.

We have implemented the variational formulations of eqs. (2) and (5) in the FEMJava platform (see Eyheramendy \& Oudin 2007, Eyheramendy, Lejeunes, Saad, \& Zhang 2014), developed in our laboratory, which allows us to used the same variationnal formations and material behavior wih both FE and IGA and thus facilitates numerical comparisons.

\section{SOME NUMERICAL SIMULATIONS}

\subsection{Hyperelastic Cook's "membrane”}

We consider the geometry and the boundary conditions defined from figure 2 . For this test we have used a plane strain formulation ${ }^{5}$ with the following hybrid energy:

$$
\rho_{0} \varphi=\frac{\mu}{2}\left(I_{1}(\overline{\mathbf{B}})-3\right)-k_{v} \exp \frac{q}{k_{v}}+q+k_{v}
$$

\footnotetext{
${ }^{4}$ In our implementation, we use a standard Gauss-Legendre quadrature scheme which is certainly not computationally optimal for NURBS functions

${ }^{5}$ We should be talking about Cook's infinite block rather than Cook's membrane for this test.
} 


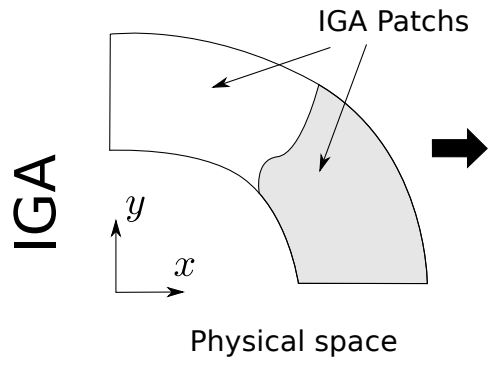

$\eta$
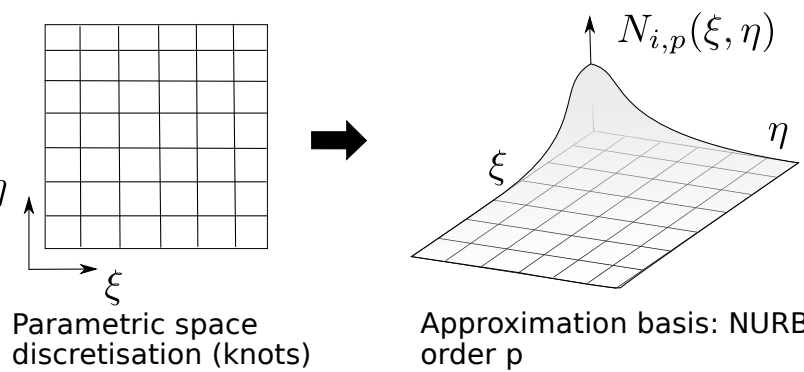

Approximation basis: NURBS order $\mathrm{p}$

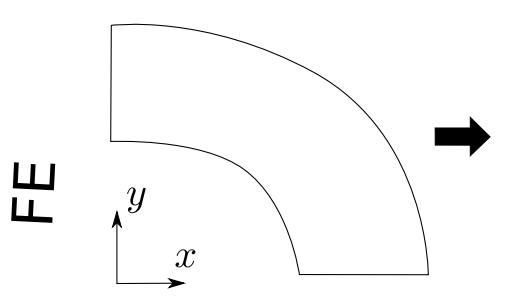

Physical space

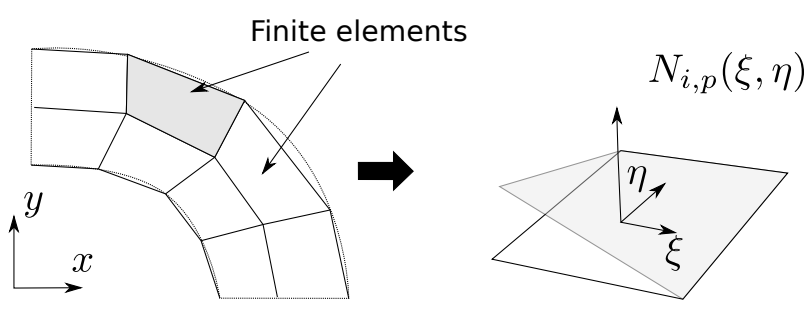

Discretisation in the physical space
Elemental parametric space and interpolation basis: Lagrange order $\mathrm{p}$

Figure 1: Comparaison of IGA and FE

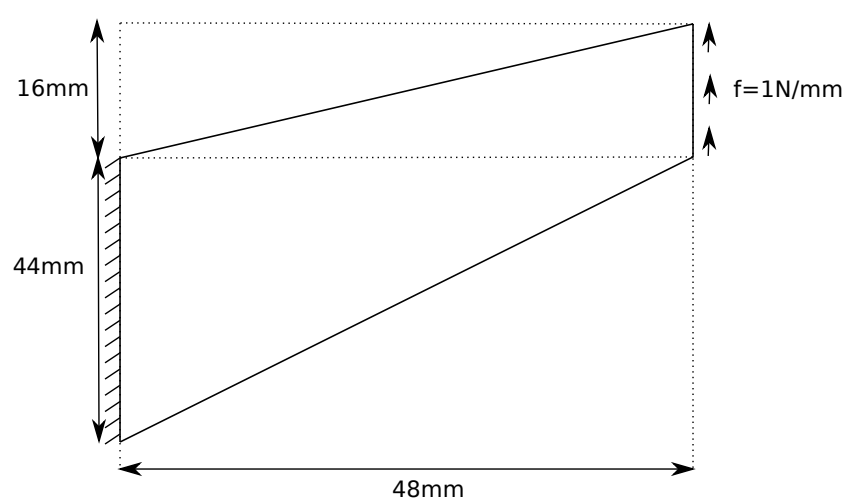

Figure 2: Geometry and boundaries condition of Cook's membrane test

where $\mu=4.62 M P a$ and $k_{v}=1500 M P a$. The applied surfacic force is $f=1 \mathrm{~N} / \mathrm{mm}$. The results are given in the figures 3 and 4 for different choices for the approximations of each field: (a) $U 3_{1}^{1} P_{2}^{1}$ corresponds to 1 order of difference between $\mathbf{u}^{h}$ and $p^{h}$ with maximum continuity and without subdivision, (b) $U 3_{1}^{1} P_{1}^{1}$ corresponds to 2 order of difference between $\mathbf{u}^{h}$ and $q^{h}$ with maximum continuity without subdivision, (c) $U 3_{1}^{2} P_{3}^{1}$ corresponds to a lower continuity for $\mathbf{u}^{h}$ than for $q^{h}$ with the same order and without subdivision, (d) $U 3_{2}^{1} P_{3}^{1}$ corresponds to a subdivision of $\mathbf{u}^{h}$ with maximum continuity and the same order for $\mathbf{u}^{h}$ and $q^{h}$. If we consider only the pressure field, we can observe that the first approximation is unstable (while the same choice leads to a stable formulation for the finite elements). Other approximations correctly describe the pressure field. Unlike finite elements, we also observe some instabilities for the kinematic field for approximations (c) and (d).

In Kadapa, Dettmer, \& Perić 2016, authors have found that approximation (d) seems stable for nearly incompressible hyperelastic Cook's membrane test. According to our results, this does not seem to be the case. Looking at the numerical test of Kadapa,

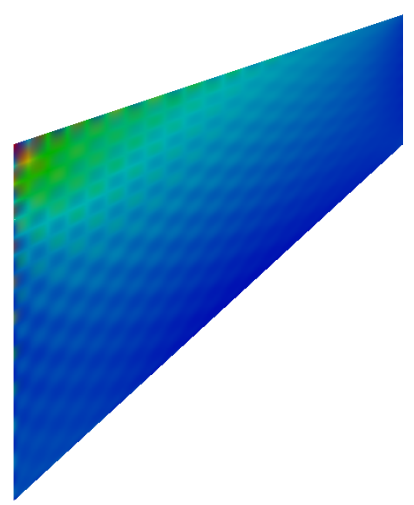

(a) IGA. $U 3_{1}^{1} P 2_{1}^{1}$

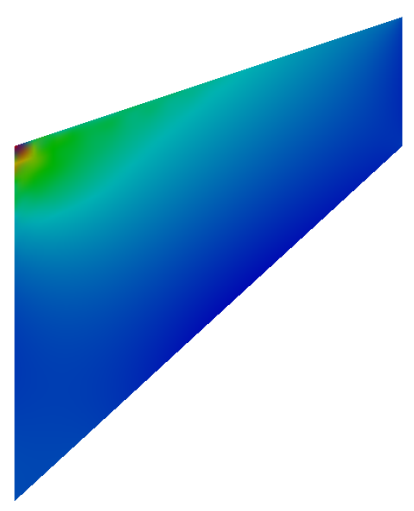

(c) IGA. $U 3_{1}^{2} P 3_{1}^{1}$

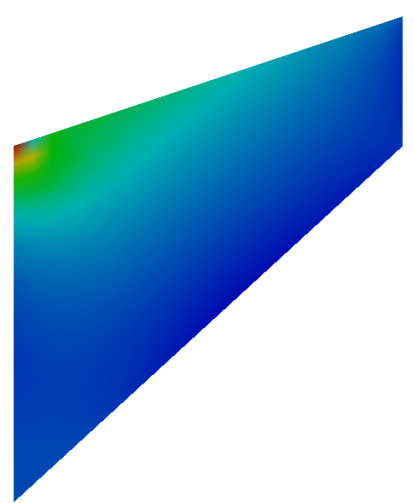

(b) IGA. $U 3_{1}^{1} P 1_{1}^{1}$

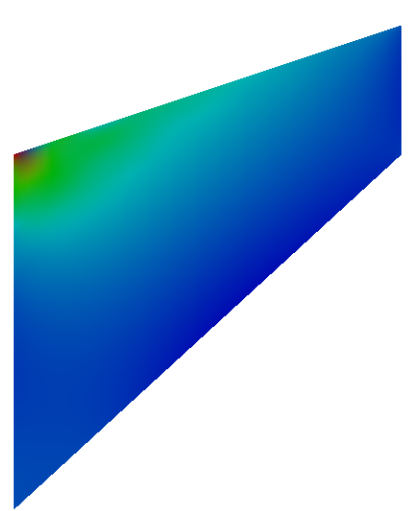

(d) IGA. $U 3_{2}^{1} P 3_{1}^{1}$
Figure 3: Cook's membrane test: Pressure field, $q^{h}$, with the same scale for isocolors in the undeformed configuation.

Dettmer, \& Perić 2016, the force value chosen by the authors leads to small strains and therefore they do not observe instabilities on the kinematic field. We obtained the same results for small or moderate strains however for large strains we observe instabilities. Finally, only the approximation (b) seems to be stable. 


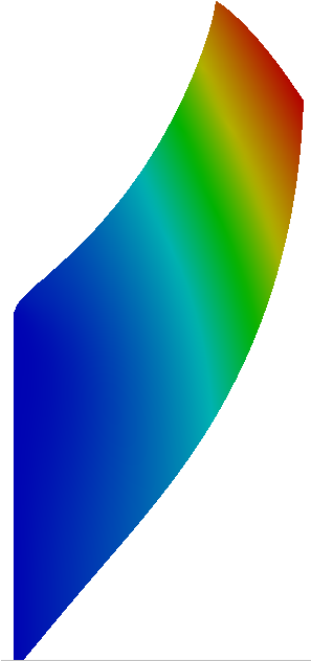

(a) $U 3_{1}^{1} P 2_{1}^{1}$

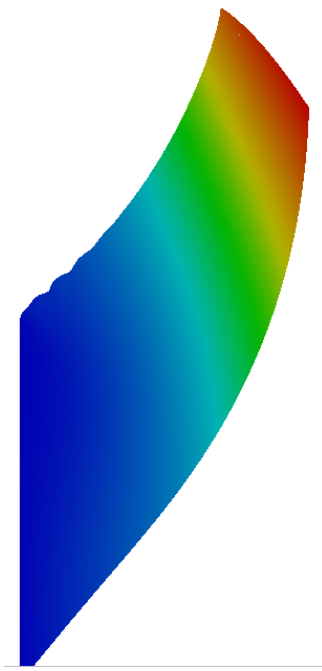

(c) IGA. $U 3_{1}^{2} P 3_{1}^{1}$

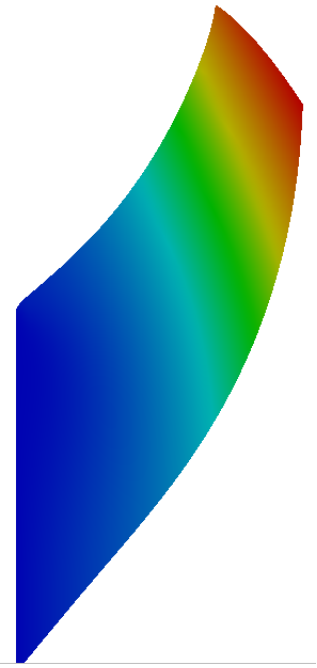

(b) IGA. $U 3_{1}^{1} P 1_{1}^{1}$

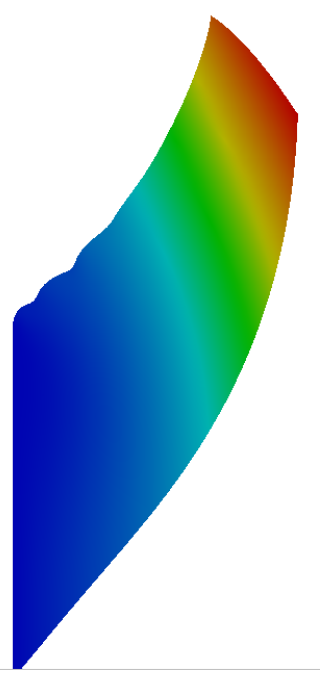

(d) IGA. $U 3_{2}^{1} P 3_{1}^{1}$
Figure 4: Cook's membrane test: Vertical componant of the kinematic field, $\mathbf{u}^{h}$, with the same scale for isocolors in the deformed configuation.

To our knowledge this results has not yet been discussed in the literature.

\subsection{Compression of a rubber block}

In this test, we consider a compression of a rubber block. The geometry and the boundary conditions are defined in figure 5. For reasons of symmetry, we only consider a quarter of the block (an eighth could be enough). The behavior and the material parameters are the same as for the previous test. We apply a very strong compression: the displacement corresponds to $50 \%$ of the height of the block. This loading generally leads to convergency problems with Finite Elements: the maximum displacement actually achieved decreases as the mesh is refine. This is due to the strong localisation of the strain (and stress) in the corner of the block (inside an element). We can therefore

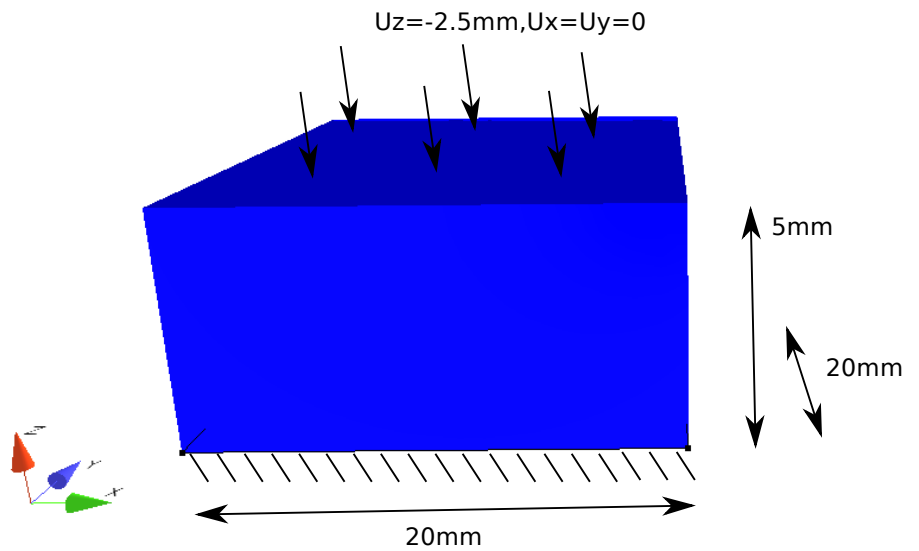

Figure 5: Geometry and boundary conditions for the compression of a rubber block test

ask ourselves the question of the relevance of the isogeometric analysis which introduce a wider geometric support for approximation functions than finite elements.

Figure 6 shows the results obtained with $\mathrm{FE}$ and IGA. The mesh size is identical for all models and we have used the stable approximation defined above for IGA. It can be observed that IGA (with different orders) allows higher compression values to be achieved than with finite elements.

\subsection{A thermoelastic case: adiabatic extension of a cracked rubber band}

For this example, we consider the following hybrid energy:

$$
\begin{aligned}
& \rho_{0} \varphi=\frac{\mu}{2} \frac{\Theta}{\Theta_{0}}\left(I_{1}(\overline{\mathbf{B}})-3\right)+C_{0}\left(\Theta-\Theta_{0}-\Theta \log \left(\frac{\Theta}{\Theta_{0}}\right)\right) \\
& -C_{1} \frac{\left(\Theta-\Theta_{0}\right)^{2}}{2 \Theta_{0}}-k_{v} \exp \frac{q}{k_{v}}+q+k_{v}
\end{aligned}
$$

where $C_{0}, C_{1}$ are material parameters that define a linear thermal dependance of the isobaric heat capacity. The 3D geometry and the boundary conditions are described at figure 7 . This test corresponds to a strecthing of a rubber band with two cracks. Adiabatic conditions are assumed for all boundaries. The entropic behavior should leads for this test to an increase of temperature excepting for small stretches where the thermoelastic inversion phenomena (competition between thermal dilatation and entropic elasticity) takes place and leads to a decrease of temperature. For this test, the material parameters are chosen such as: $\mu=4.62 e^{6} \mathrm{~Pa}, k_{v}=1500 e^{6} \mathrm{~Pa}, C_{0}=8.5 e^{5} \mathrm{~J} / \mathrm{m}^{3} / \mathrm{K}$, $C_{1}=5.9 e^{5} \mathrm{~J} / \mathrm{m}^{3} / K, \alpha=6.6 e^{-4} K^{-1}$. We adopt a standard isotropic Fourier law for the heat flux, such as $\mathbf{Q}=-k_{t} \nabla_{X} \Theta$, with $k_{t}=0.15 \mathrm{~W} / \mathrm{m} / \mathrm{K}$. 


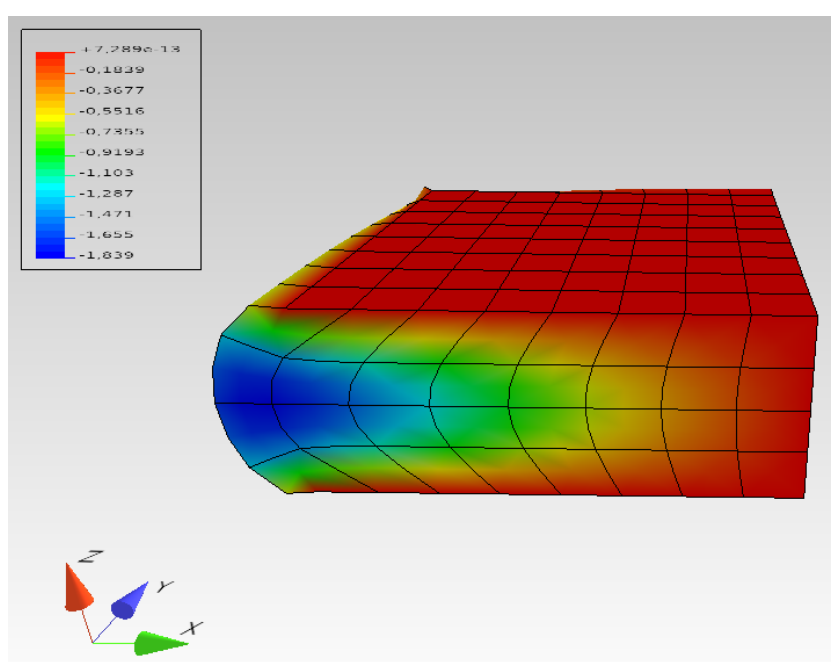

(a) E.F. $U 2 P 1,30 \%$ of maximal displacement

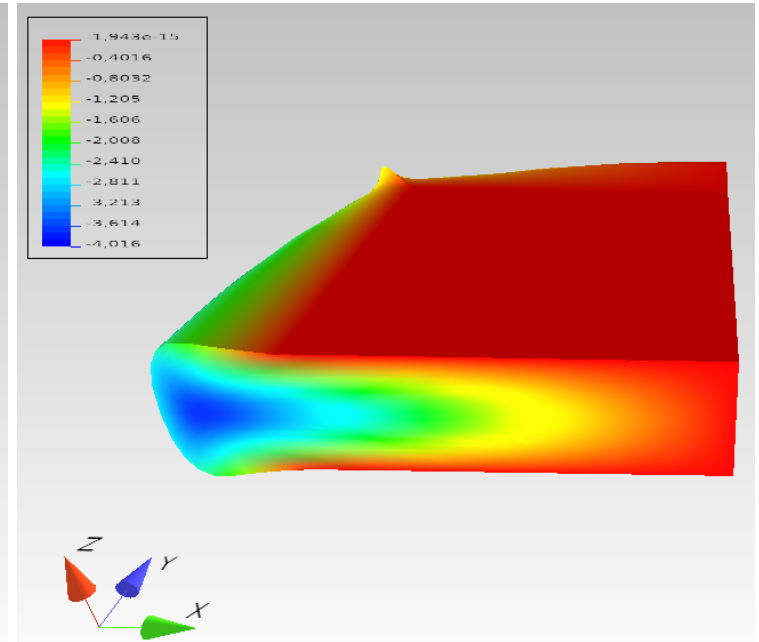

(b) IGA $U 2{ }_{1}^{1} P 0_{1}^{1}, 67 \%$ of maximal displacement

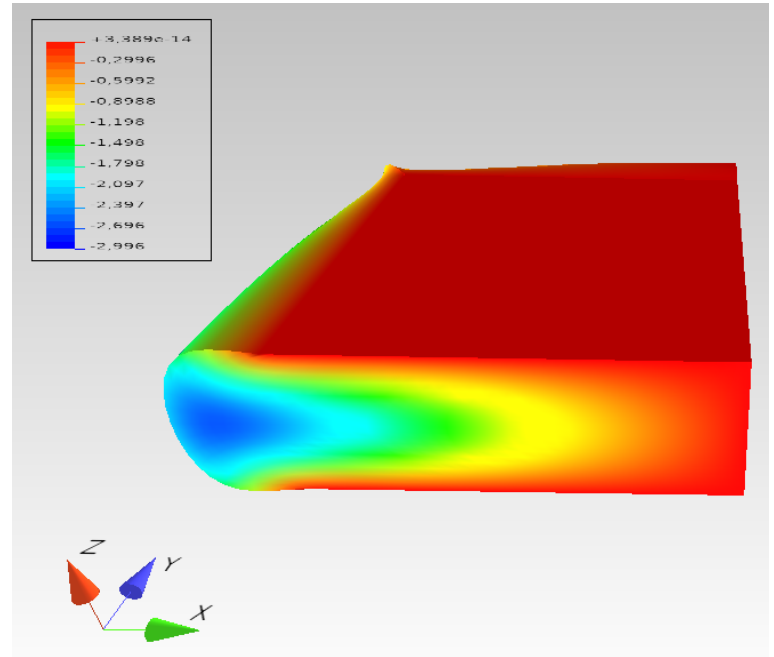

(c) IGA $U 3{ }_{1}^{1} P 1_{1}^{1}, 46 \%$ of maximal displacement

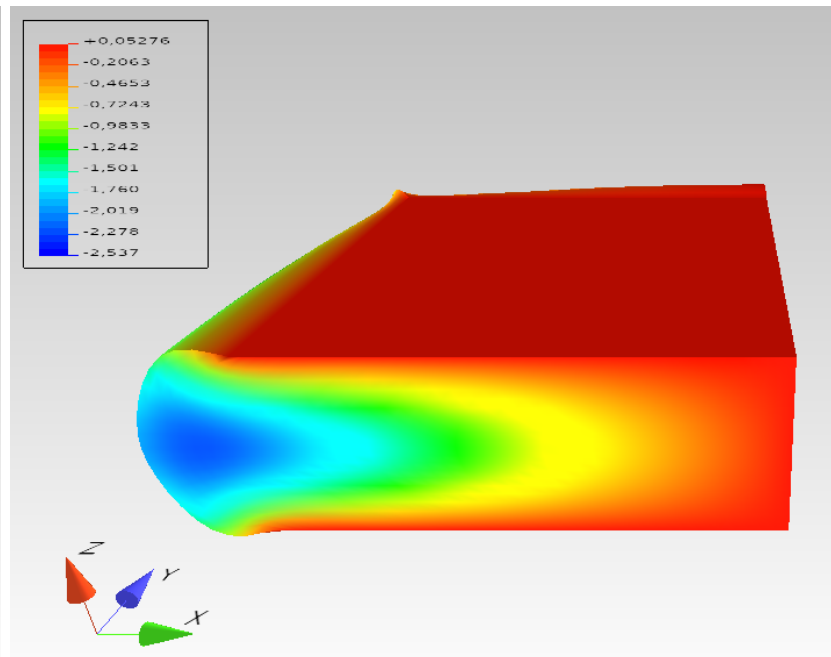

(d) IGA $U 4_{1}^{1} P 2_{1}^{1}, 46 \%$ of maximal displacement

Figure 6: Compression of a rubber block: X componant, $u_{x}$, of the kinematic field for the same mesh size $(h=1.25 \mathrm{~mm})$.

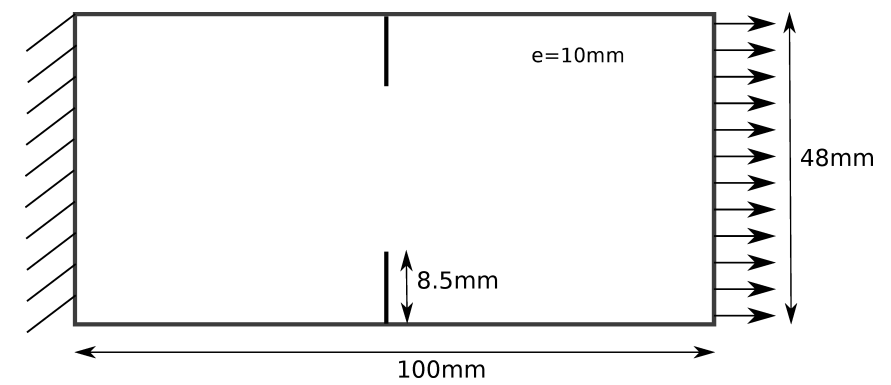

Figure 7: Cracked rubber band: geometry and boundary conditions (thickness is $10 \mathrm{~mm}$ )

Using the same formulation and the same material behavior, we can compute the solution of this test both with FE and IGA. For FE, we use hexadric elements with a quadratic kinematic, a quadratic temperature and a linear (discontinuous between elements) pressure. For IGA we use a $U 3_{1}^{1} T 3_{1}^{1} P 1_{1}^{1}$ approximation. Figures 8 and 9 show the results obtained for the temperature field (same isoscale and same stretching values for FE and IGA). We can observe that the solu- tion is qualitatively similar with FE and IGA, however the mesh is coarser for IGA: there is five times less degree of freedom for IGA than for FE for this test. Other numerical tests need to be performed to quantify the numerical performance of IGA for nonlinear thermo-elastic tests. Compared to finite elements, as with purely hyperelastic formulations, IGA offers more possibilities in the choice of approximation spaces. 

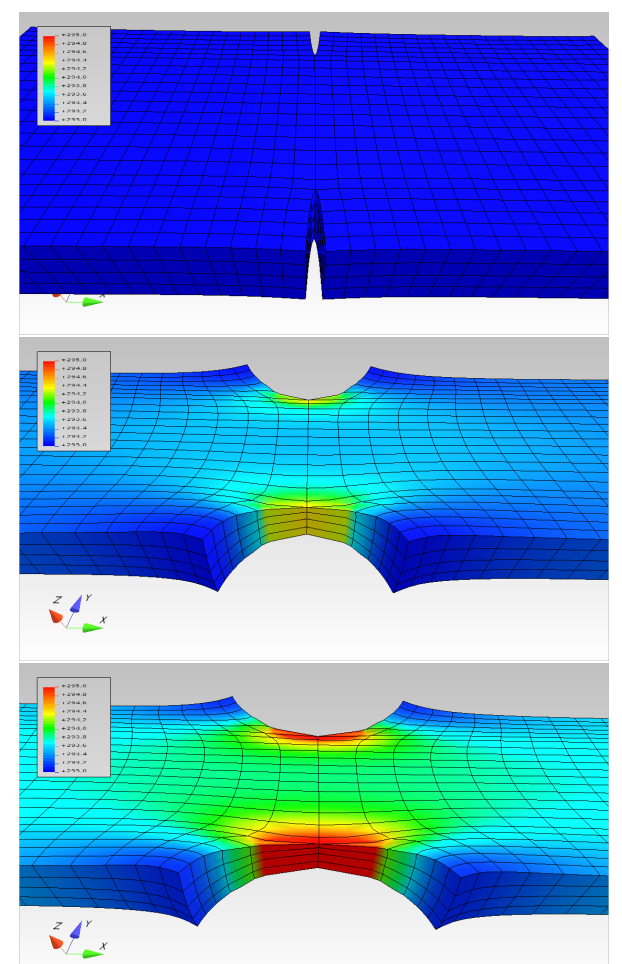

Figure 8: Temperature field for different stretches, FE solution (U2T2P1)
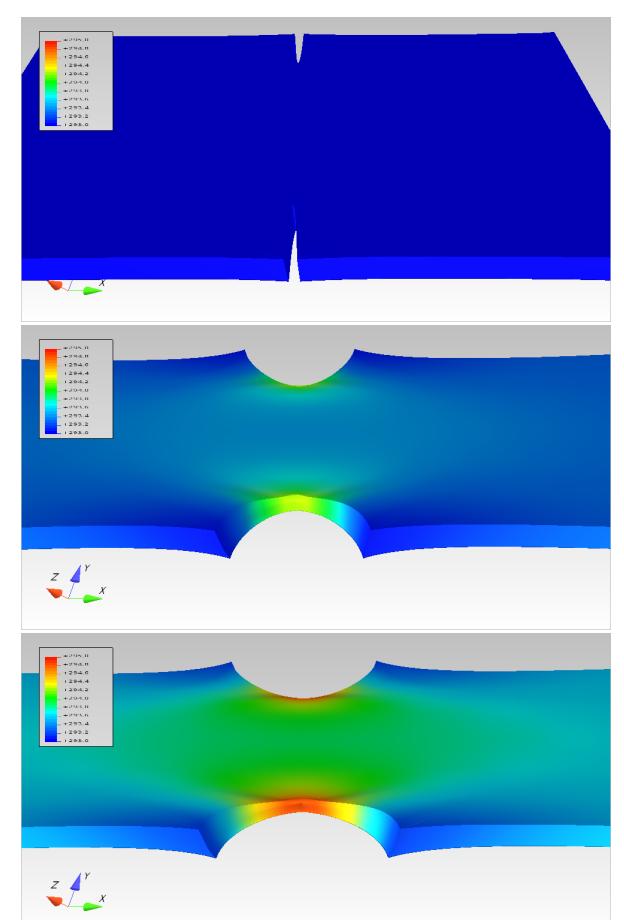

Figure 9: Temperature field for different stretches, IGA solution $\left(U 3_{1}^{1} T 3_{1}^{1} P 1_{1}^{1}\right)$

\section{CONCLUSION}

Altough isogeometric analysis is not yet widely used for simulation of complex rubber structures, this method can be numerically efficient in the context of nearly-incompressible materials. From simple examples, we have shown that we can build a stable mixed, $(\mathbf{u}, p)$, formulation in the context of hyperelasticity by playing with the order of approximation of the kinematic and pressure fields. Unlike finite elements, the pressure field should be 2 orders less than the kinematic field to obtain a stable formulation at finite strain. From the 3D compression test, we show that with the same mesh size, IGA can achieve a higher level of deformation in compression. Using the hybrid energy concept and a multifield formulation, the extension to thermo-mechanical cases is straightforward. As in the hyperelastic case, from a simple example, we illustrate the fact that IGA can achieve great numerical performances with less degrees of freedom than FE.

\section{REFERENCES}

Buffa, A. abd de Falco, C. \& G. Sangalli (2010). Isogeometric analysis: Stable elements for the $2 \mathrm{~d}$ stokes equation. International Journal for Numerical Methods in Fluids 65, 14071422.

Elguedj, T. \& T. J. R. Hughes (2014). Isogeometric analysis of nearly incompressible large strain plasticity. Computer Methods in Applied Mechanics and Engineering 268, 388-416.

Eyheramendy, D., S. Lejeunes, R. Saad, \& L. Zhang (2014). Advances in symbolic and numerical approaches in computational mechanics. In B. H. V. Topping and P. Iványi (Eds.), Computational Methods for Engineering Technology, Chapter 3, pp. 61-88. Saxe-Coburg Publications.

Eyheramendy, D. \& F. Oudin (2007). Advanced object-oriented techniques for coupled multiphysics (3th chapter). In T. B. H. V. (Ed.), Civil engineering computations: tools and techniques, Computational Science, Engineering \& Technology Series, pp. 37-60. Saxe-Cobourg Publications. ISSN 17593158 OR 23 OR 23.

Flory, P. J. (1961). Thermodynamic relations for highly elastic materials. Transactions of the Faraday Society 57, 829-838.

Hughes, T., J. Cottrell, \& Y. Bazilevs (2009). Isogeometric Analysis. Wyley.

Hughes, T. J. R., J. A. Cottrell, \& Y. Bazilevs (2005). Isogeometric analysis: Cad, finite elements, nurbs, exact geometry and mesh refinement. Computer Methods in Applied Mechanics and Engineering 194, 4135-4195.

Kadapa, C., W. G. Dettmer, \& D. Perić (2016). Subdivision based mixed methods for isogeometric analysis of linear and nonlinear nearly incompressible materials. Computer Methods in Applied Mechanics and Engineering 305, 241-270.

Lejeunes, S. \& D. Eyheramendy (2018, May). Hybrid free energy approach for nearly incompressible behaviors at finite strain. Continuum Mechanics and Thermodynamics.

Miehe, C. (1995). Entropic thermoelasticity at finite strains. aspects of the formulation and numerical implementation. Computer Methods in Applied Mechanics and Engineering 120(3), $243-269$.

Nielsen, P. N., A. R. Gersborg, J. Gravesen, \& N. L. Pedersen (2011). Discretizations in isogeometric analysis of navier-stokes flow. Computer Methods in Applied Mechanics and Engineering 200(45), 3242 - 3253.

Zhang, L. (2016). An isogeometric analysis approach for coupled multi-field problems at large strain. Ph. D. thesis, Ecole Centrale Marseille. 